\title{
Application of biomimetic HPLC to estimate in vivo behavior of early drug discovery compounds
}

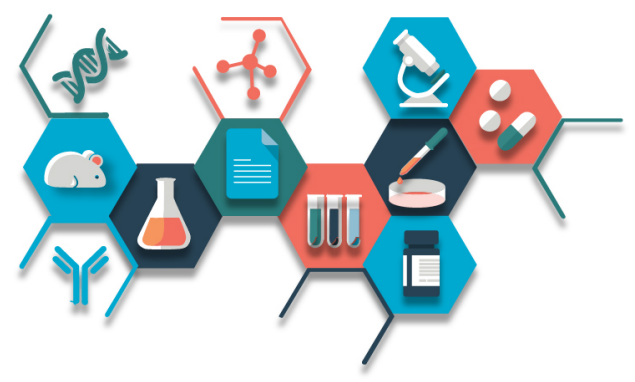

\author{
Klara L Valko*,1,2 \\ ${ }^{1}$ Bio-Mimetic Chromatography Ltd, Unit 5B Business \& Technology Centre, Bessemer Drive, Stevenage, SG1 2DX, UK \\ ${ }^{2}$ UCL School of Pharmacy, 29-39 Brunswick Square, London, WC1N 1AX, UK \\ *Author for correspondence: k.valko@ucl.ac.uk
}

Characterizing the properties of large numbers of compounds and estimating their potential absorption, distribution, metabolism and elimination properties are important early stages in the process of drug discovery and help to reduce later stage attrition. The chromatographic separation principles using stationary phases that contain proteins and phospholipids are more suitable for compound characterization and estimation of the pharmacokinetic properties than the traditional octanol/water partition coefficient. This technology, when standardized, enables the prediction of in vivo behavior and the selection of compounds with the best potential, thus reducing the number of animal experiments. Chromatography may be involved more widely in the future to measure kinetic aspects of compounds' binding to proteins and receptors which would enable designing compounds that require a lower frequency of doses and have more predictable pharmacokinetic profiles.

Lay abstract: The measurement of the molecular properties of potential drug molecules is now an established component of modern drug design strategies. Chromatographic separation now plays an essential role in this process in the estimation of pharmacokinetic properties and may be extended in the future to protein and receptor binding kinetics. These strategies will also offer cost savings and reduce the need for animal experiments.

First draft submitted: 15 January 2019; Accepted for publication: 4 March 2019; Published online:

26 April 2019

Keywords: absorption $\bullet$ attrition reduction $\bullet$ biomimetic chromatography $\bullet$ chromatographic lipophilicity $\bullet$ distribution • drug efficiency $\bullet$ human serum albumin binding $\bullet$ immobilized artificial membrane chromatography • metabolism and elimination prediction • phospholipid binding • volume of distribution

Medicinal chemists face multidisciplinal and multivariable problems in designing drug molecules that can efficaciously reduce or cure a pathological condition; medicinal chemists will seek the smallest possible dose with minimal side effects [1-4], yet they are often plagued with too much in vitro and in vivo data on the compounds to make a decision. The latest studies show that the most significant problems with the late-stage attrition of discovery molecules are those regarding safety and efficacy. The safety issue has been related to lipophilicity [5-8] that may cause the compound to bind to unwanted targets. The root cause of these problems is partially dominated by the compound's binding or partitioning into tissue which contains membrane phospholipids. When this happens, the drug is sequestered into nonspecific phospholipid binding sites and is unavailable as a free drug near target receptors [9-11].

This offers an explanation as to why lipophilicity is one of the most often used properties that can be related to a compound's in vivo distribution behavior, toxicity and in general its bioaccumulation [12,13]. A drug's binding to proteins may also involve lipophilic attractions and its partition to tissues is also related to its lipophilicity [14]. Since the early work of Corwin Hansch, the octanol/water partition coefficients have been used as a measure of the loss in the concentration of drug molecule that appears at the site of action relative to the administered dose. The octanol/water partition system appeared to mimic very well the compound's nonspecific binding to proteins and lipids [15]. 
In this perspective article, the applicability and the advantages of using chromatographic separation principles for the characterization of physicochemical and biomimetic binding properties of compounds are highlighted. It is emphasized that octanol/water partition coefficients are not suitable for the estimation of in vivo distribution of molecules, especially when they are charged at physiological $\mathrm{pH}$. Chromatography using protein and phospholipid stationary phases is more suitable for estimating a compound's distribution in the body. When applied at an early stage of the drug discovery process, this technology enables the characterization of many discovery compounds and the prediction of their in vivo behavior at a much earlier stage. The latest developments have revealed that the technique can be used for peptides and miniproteins, for which the traditional methodologies have been failing $[16,17]$.

The perspective of using chromatographic biomimetic properties in early drug discovery will help to reduce later stage attrition of candidate molecules due to disadvantageous absorption, distribution, metabolism and elimination (ADME) properties. Chromatography may be involved more widely to measure the kinetic aspects of compound's binding to proteins and receptors at early stages of the drug discovery process that would enable the design of compounds with less frequent doses and more predictable pharmacokinetic profiles [18-20].

\section{Principles of the biomimetic HPLC methods \\ C-18 lipophilicity using reversed phase retention}

It has been recognized and elegantly described by the solvophobic theory [21,22] that retention in aqueous reversed phase chromatography is governed by lipophilicity or more exactly by hydrophobicity. This means that the chromatographic retention times are directly related to the compound's dynamic distribution coefficient between the stationary and the mobile phases. The chromatographic method has numerous advantages over the traditional method of measuring liquid/liquid partition coefficients. A much smaller amount of compound is needed for the reversed phase retention measurements and small impurities do not alter the results as they are separated from the main component. The retention time measurements are inherently more precise than the concentration determination process for the shake flask (octanol/water) method.

The partition coefficient $(\mathrm{K})$ of a compound between the stationary and the mobile phase can be expressed by using the retention factor to express the compound's concentration in the stationary and mobile phase in moles/volume.

Equation 1 shows the relationship between the logarithmic retention factor and the logarithmic partition coefficient of a compound in the chromatographic stationary and mobile phases.

$$
\log K=\log k+\log \left(\frac{V_{s}}{V_{m}}\right)
$$

$\mathrm{V}_{\mathrm{s}}$ and $\mathrm{V}_{\mathrm{m}}$ are the volumes of the stationary and mobile phases, respectively. Equation 1 shows that the $\log \mathrm{k}$ values are in a linear relationship with the $\log \mathrm{K}$ values which are the partition coefficients. The intercept is the so-called phase ratio, the proportion of the volumes of the stationary phase and mobile phase in the column. This is a constant value for each HPLC column that is difficult to determine accurately. Therefore, it is suggested to calibrate the chromatographic retention times of compounds by using their respective distribution coefficients, preferably containing the same phases. For the analysis of compounds with a wide range of lipophilicities, it is necessary to change the mobile phase composition and mix the aqueous buffer with various concentrations of a miscible organic solvent (usually acetonitrile or methanol). Higher organic phase concentrations in the mobile phase will reduce the retention of more lipophilic compounds. The relationship between the reversed phase retention and the concentration of the organic phase in the mobile phase has been analyzed in detail [23]. A linear relationship can be expected between the $\log \mathrm{k}$ values and the concentration of the organic phase in the mobile phase in reversed phase chromatography. The question then arose of how the retention factors could be extrapolated to a single scale for comparison. Equations 2 and 3 describe the linear or quadratic relationships between the retention factor and the organic phase concentration as a volume percentage $(\varphi)$ in the mobile phase, which may provide a solution.

$$
\log k=a^{*} \varphi+b
$$




$$
\log k=c^{*} \varphi+d^{*} \varphi^{2}+e
$$

There is a quasilinear portion of the function for which we can establish the $a$ and $b$ constants by carrying out the $\log \mathrm{k}$ measurements using three to five different organic phase concentrations $(\varphi)$ in the mobile phase. The $b$ constant is the extrapolated log $\mathrm{k}$ value to zero organic phase concentration, in other words neat water (buffer), often referred to as $\log \mathrm{k}_{0}$ or $\log \mathrm{k}_{\mathrm{w}}$.

The approach described above raises some potential questions; it is possible that the straight line is valid only for a limited range of organic phase concentrations, and therefore other equations and numerical methods have been suggested [24,25] to derive $\log \mathrm{k}_{\mathrm{w}}$ values. The straight lines of the compounds may cross each other. This has been observed in the past and the use of slope values as the second variable in a correlation to octanol/water coefficient has been suggested [26].

To avoid several isocratic measurements, fast gradient reversed phase chromatography has been proposed to derive the chromatographic hydrophobicity index (CHI) that approximates the organic phase concentration in the mobile phase when the compound elutes in gradient mode [27]. When a fast-organic phase gradient is applied on a reversed phase column, the compounds do not move in the chromatographic system until the appropriate organic phase concentration reaches the column and starts eluting the compound practically with the dead time. Acetonitrile gradient reversed phase chromatography has been introduced to measure lipophilicity of drug discovery compounds and has been compared with octanol/water lipophilicity [28]. It was found that the isocratically determined organic phase concentration that resulted in the retention factor $1\left(\phi_{0}\right)$ showed a linear relationship with the fast-gradient retention times of the compounds. The CHI has been derived from this linear relationship. The CHI value approximates the organic phase concentration in the mobile phase by which the compound's retention time is double that of the dead time, in other words retention factor $(\mathrm{k})$ equal to 1 and $\log \mathrm{k}=0$. In this way, there is no need to carry out several isocratic measurements and the extrapolation to zero organic phase concentration in the mobile phase or a backward extrapolation to a certain organic phase concentration. The CHI values range from 0 to 100 (for very hydrophilic or lipophilic molecules, it can go below zero or above 100, respectively). The higher the value, the more lipophilic the compound is as a higher organic phase concentration is needed in the mobile phase to elute the compound from the nonpolar stationary phase. In order to help medicinal chemists to develop structure lipophilicity relationships, we can project the $\mathrm{CHI}$ values to the octanol/water $\log \mathrm{D}$ scale to obtain CHIlog D or Chromlog $\mathrm{D}$ as described by equations 4 and 5 .

$$
C H I l o g D=0.054 * C H I-1.467
$$

$$
\text { Chromlog } D=0.088 C H I-2
$$

Both the CHIlogD and ChromlogD scale are linear with the $\mathrm{CHI}$ scale. CHIlogD was derived from 98 diverse compounds with $\mathrm{CHI}$ and octanol/water $\log \mathrm{D}$ values, while the ChromlogD scale was derived from over 40,000 compounds with a calculated $\log \mathrm{D}$ and then related to $\mathrm{CHI}$. ChromlogD gives approximately a 2-log unit higher lipophilicity value than the octanol/water $\log \mathrm{D}$ for compounds having three to five $\log \mathrm{D}$ values in the octanol/water system.

The chromatographic lipophilicity that is determined using the C-18 stationary phase with acetonitrile as an organic mobile phase component, does not provide an octanol/water type lipophilicity. It provides a dynamic partition coefficient of a compound between the hydro-organic mobile phase and C-18. Which provide a more structured environment than a liquid solvent, due to the bonding of alkyl chains on a silica surface. When comparing the two types of lipophilicity using the Abraham solvation equation approach [29], it was found that the major difference is the sensitivity toward H-bond acidity. H-bond donor compounds partition into octanol as it has an $\mathrm{H}$-bond acceptor $\mathrm{OH}$ group, unlike the $\mathrm{C}-18$ stationary phase that does not accept $\mathrm{H}$-bonding. Figure $1 \mathrm{~A}$ and $\mathrm{B}$ shows the relationship between the $\log \mathrm{P}$ and $\mathrm{CHI}$ values without and with the $\mathrm{H}$-bond acidity parameter. It is important to observe that the lipophilicity of a compound expressed by a liquid-liquid partition coefficient is not the same as the chromatographic lipophilicity using reversed phase (C-18) stationary phase. The two solvent systems are very different (octanol and a C-18 alkyl chain), as are the partition processes as the chromatographic 

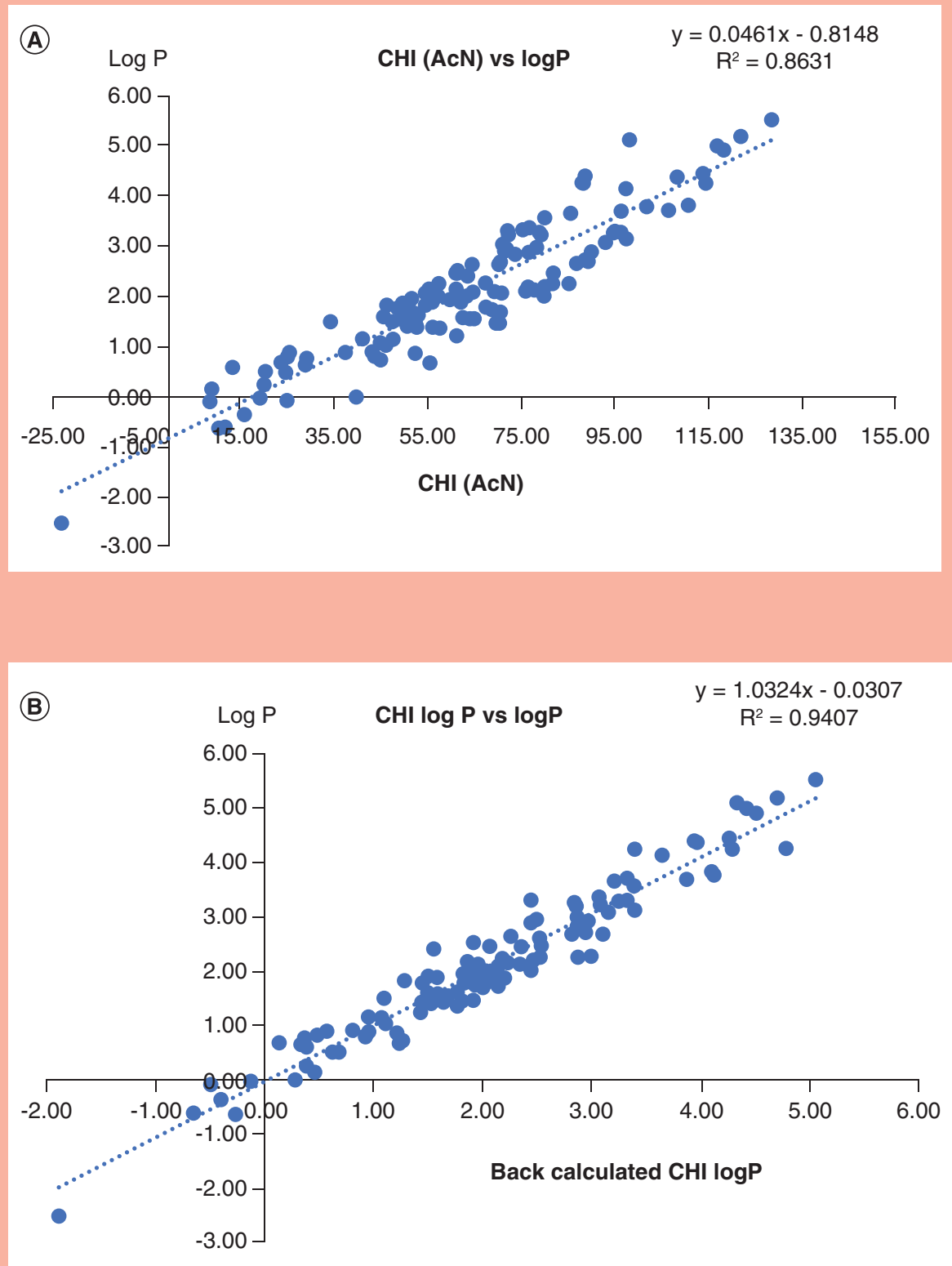

Figure 1. The relationship between $\log \mathrm{P}$ and $\mathrm{CHI}$ without $(\mathrm{A})$ and with the $\mathrm{H}$-bond acidity parameter (B).

partition takes place on a large surface and therefore is more entropy driven. Methanol as an organic modifier would be more similar to octanol, but it produces a parabolic relationship between $\log \mathrm{P}$ as shown in Figure 2 .

When amphiphilic large molecules are investigated in a liquid-liquid partition system, they tend to accumulate in the solvent interface. Thus, the concentration ratio in the two phases as a partition coefficient can be misleading.

The chromatographic distribution process is a different but better model for in vivo distribution processes as they are dynamic and develop on large surfaces (stationary phase and protein/phospholipid membrane).

In order to reveal the acid/base character of drug discovery compounds, which plays a very important role in the in vivo distribution, the reversed phase gradient retention times can be measured using acidic, neutral and basic buffers. Positively charged compounds have higher lipophilicity at high $\mathrm{pHs}$, while negatively charged compounds have high lipophilicity at low $\mathrm{pH}$ due to their loss of charge. Compound distribution between the plasma and the tissue compartment strongly depends on the type of charge, something that will be discussed later. The $\mathrm{pH}$ and $\mathrm{pKa}$ changes during reversed phase gradient elution have been thoroughly investigated $[18,30,31,32]$ and, 


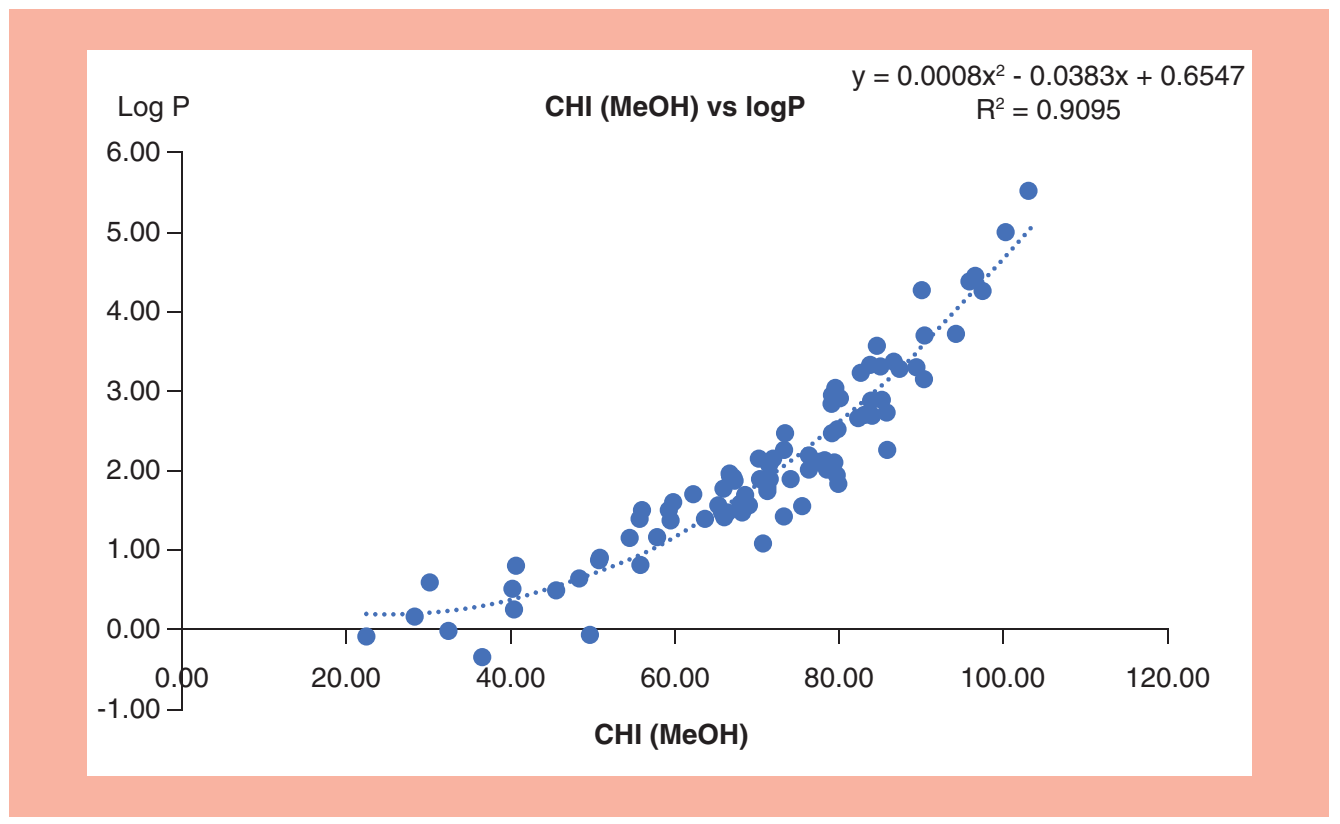

Figure 2. The plot of chromatographic hydrophobicity index (MeOH) and log $\mathbf{P}$ for 86 divers compounds.

moreover, a method has been developed to estimate the pKa of compounds by using two gradient reversed phase chromatographic runs [33].

\section{Protein \& immobilized artificial membrane biomimetic stationary phases}

When the major body components are applied as stationary phases, it would reveal a compound's interactions with these particular proteins and phospholipids.

Human serum albumin (HSA) and AGP can be chemically bonded to a silica support, and they are commercially available HPLC stationary phases which were developed for chiral separations. The chemical bonding of the proteins is carried out in a way as to not to distort the natural binding sites of the proteins. It was essential to prove that the chromatographic retention data obtained from these protein phases truly revealed the compound's binding to these proteins in the body. Therefore, a thorough validation was carried out using known drugs with HSA and AGP-binding data $[15,34]$. The plots of the HSA and AGP binding obtained by chromatography, equilibrium dialysis and ultrafiltration are shown in Figure 3.

Both plots show relatively large discrepancies between the HPLC data and the binding data obtained by the equilibrium dialysis and ultrafiltration methods. In the case of HSA binding, it was compared with total human plasma protein binding that contains other proteins, not just HSA. Kratochwill et al. [35] showed that chromatographic binding data gave a better correlation with equilibrium dialysis data when only albumin was used and not plasma. The AGP binding was slightly stronger using HPLC for basic compounds, but the neutral and acidic compounds showed a good correlation. The biggest advantage of using the HPLC method is that the rank order of compound binding is very reproducible, especially in the strong binding range where other methodologies lose their sensitivity. The other advantage is that chemists can build structure binding relationships easily with the individual proteins in the plasma so that in the disease state when albumin and glycoprotein concentration in the plasma can change in patients, the total binding can be estimated.

The immobilized artificial membrane (IAM) phase was developed by Pidgeon et al. [36] with the aim to emulate the lipid membrane on a solid surface. The commercially available IAM.PC.DD2 column manufactured by Regis Technologies Inc. contains the phosphatidylcholine head group and mimics the phospholipid membrane monolayer. A compound's partitioning to this stationary phase reveals the phospholipid affinity of the compound which is necessary to achieve permeability. However, very strong affinity reduces the compound's permeability through the membrane bilayer as the compound prefers to stay inside the membrane. These compounds usually cause membrane disruption or phospholipid metabolism disorder (phospholipidosis) in the cells [37-40]. 


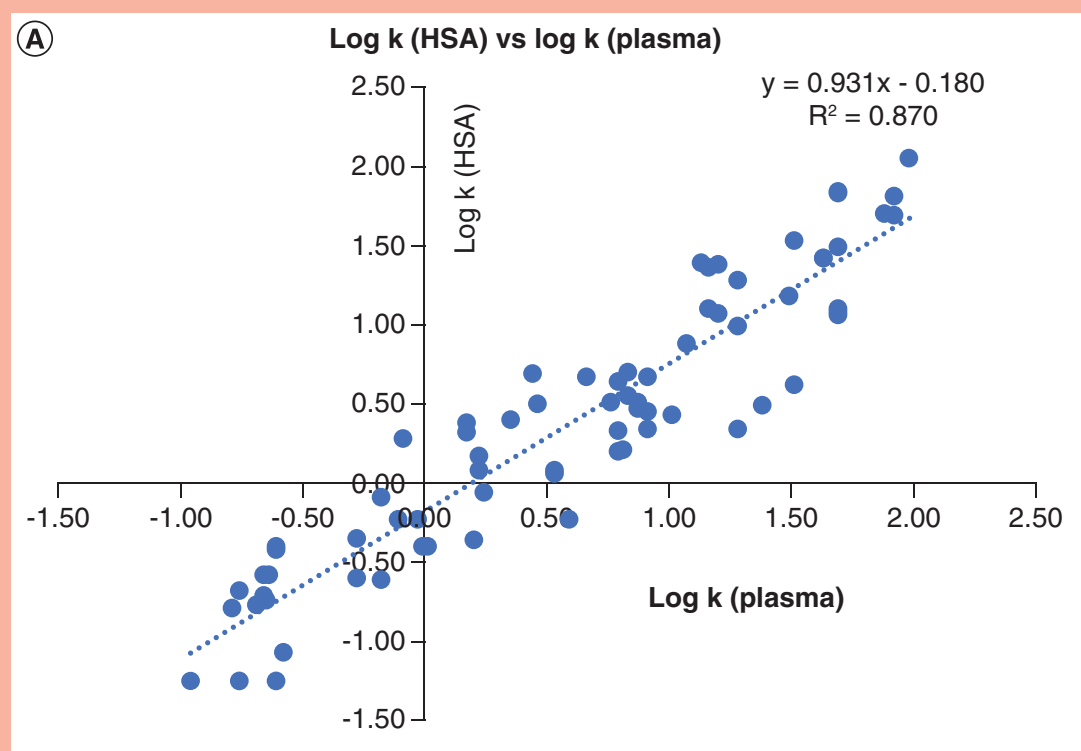

(B)

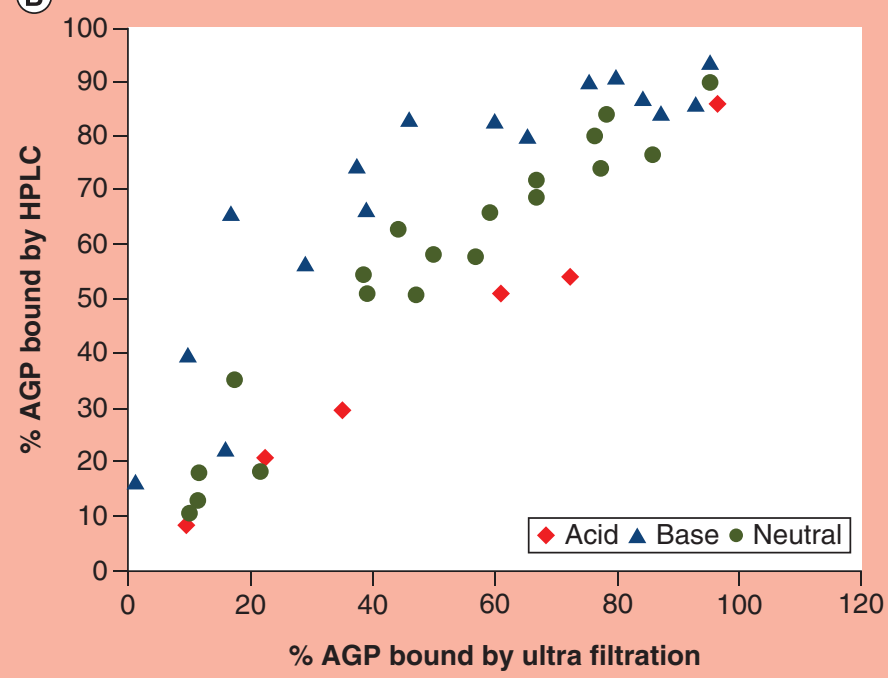

Figure 3. The plots of HSA binding obtained by HSA-HPLC (log k [HSA]) and equilibrium dialysis (log $k$ [plasma]) (A) and AGP-HPLC and ultrafiltration (B).

HPLC: High-performance liquid chromatography; HSA: Human serum albumin.

Typical experimental conditions to measure biomimetic HPLC-based protein and phospholipid binding have been described [9].

\section{Standardization \& data transformation}

The biomimetic HPLC measurements provide information about a compound's interaction with the actual protein and phosphatydil choline using chromatographic retention data. As the chromatographic retention factor depends on the experimental conditions, it is important to calibrate the chromatographic system by measuring the retention data for a set of compounds for which the binding data are available from other methodologies such as equilibrium dialysis, ultrafiltration or capillary electrophoresis frontal analysis [41]. In this way, the obtained data will be comparable between laboratories and reproducibility is improved. Checking the protein stationary phase to make sure no denaturation or irreversible binding has occurred during the measurements is also essential. The calibration plot is checked before and after each 96-well plate sample run. It is interesting to note that as protein and 
Table 1. Abbreviations used throughout the text.

\begin{tabular}{|c|c|}
\hline Log $\mathrm{k}$ & Logarithmic retention factor \\
\hline IAM & Immobilized artificial membrane (HPLC stationary phase) \\
\hline HSA & Human serum albumin \\
\hline AGP & $\alpha$-1-acid-glycoprotein \\
\hline PPB & Total plasma protein binding \\
\hline $\mathrm{PFI}$ & Property Forecast Index \\
\hline Vdu & Unbound volume of distribution \\
\hline Vdss & Steady state volume of distribution (dose/plasma concentration) \\
\hline BTB & Brain tissue binding \\
\hline PPB & Plasma protein binding \\
\hline $\mathrm{fu}$ & Unbound fraction in plasma \\
\hline $\mathrm{DE}$ & Drug efficiency $(100 \times$ free biophase concentration/dose) \\
\hline DEmax & Drug efficiency measured by IAM binding and protein binding by HPLC ( $100 \times$ free plasma concentration/dose $)$ \\
\hline DEI & Drug efficiency index, potency plus log DEmax (plc50 + log DE) \\
\hline clogP & Calculated logarithm of octanol/water partition coefficient \\
\hline $\log \mathrm{D} / \mathrm{P}$ & Logarithm of distribution/partition coefficient \\
\hline hERG & The human Ether-à-go-go-related gene, that codes for a protein known as $\mathrm{K}_{\mathrm{v}} 11.1$, the $\alpha$ subunit of a potassium ion channel. \\
\hline
\end{tabular}

phospholipid binding occurs naturally at physiological $\mathrm{pH}$, there is no need to apply mobile phases with different pHs.

The measurements are carried out using the gradient retention times of a set of eight to ten compounds with known binding and lipophilicity data. The slope and intercept values of the straight lines obtained can then be used to convert the measured retention times to binding values. It is important to note that the retention times data does not always show linear correlation with binding data; Table 1 summarizes the meaning of the abbreviations used in the equations, while Table 2 shows the equations that are used to convert retention data to actual binding values.

\section{Simple models to estimate developability}

Combining the number of aromatic rings in the molecule with the ChromlogD value gives the so-called PFI values (Property Forecast Index) [45]. The PFI values between three and six are ideal for expecting good ADME properties for drug discovery compounds. It was found on the basis of the analysis of over 40,000 compounds that, when PFI values were between three and six, the majority of the compounds had solubilities of over $200 \mu \mathrm{g} / \mathrm{ml}$, HSA binding less than $95 \%$ and $\mathrm{p} 450$ inhibition $\left(\mathrm{pIC}_{50}\right.$ ) of less than five. Compounds with lower than PFI 3 showed limited permeability in the artificial membrane permeability assay. Compounds that showed higher than six in PFI values when the neutral form Chrom $\log \mathrm{D}$ was taken into consideration showed hERG inhibition, an indication of a potential effect on the heart rhythm and promiscuous binding that was positive when the compound showed stronger than five in $\mathrm{pIC}_{50}$ values on more than five targets. Although these observations were made on large number of compounds, there are always exceptions from the rules but having a PFI value between three and six increases the probability of success for a candidate drug molecule during drug the development process.

\section{Models to estimate in vivo distribution \& drug efficiency}

Several model equations are shown in Table 2. The volume of distribution can be defined as the proportion of the dose and the steady state plasma concentration that is, in principle, proportional to the drug distribution between the plasma and the tissue compartment. It can be obtained only by using in vivo experiments. Therefore, estimating the volume of distribution early in the drug discovery process using only two biomimetic HPLC measurements is very valuable. Figure 4 shows a typical plot between the measured human volume of distribution of known drugs and the estimated values using the equation listed in Table 2. The model is based on the difference between the IAM and HSA binding of the compound. Compounds that bind more strongly to phospholipids partition more into tissues, while strong albumin binding compounds prefer staying in the plasma compartment. It was also observed [42] 
Table 2. Equation used to convert measured chromatographic data and model equations for the estimation of in vivo distribution of compounds.

\begin{tabular}{|c|c|c|}
\hline Parameter meaning & Calculations & Ref. \\
\hline $\mathrm{CHI}(\mathrm{C}-18)$ & $\begin{array}{l}\text { Chromatographic hydrophobicity index obtained on } \mathrm{C}-18 \text { column using the calibration line }(\mathrm{CHI}=\text { slope* } \\
\text { gradient retention time }+ \text { intercept })\end{array}$ & \\
\hline CHIlogD & $C H I l o g D=0.054 * C H I-1.467$ & \\
\hline ChromlogD & Chrom $\log D=0.088 * \mathrm{CHI}-2$ & \\
\hline PFI & ChromlogD + the number of aromatic rings & \\
\hline CHI (IAM) & $\mathrm{CHI}(\mathrm{IAM})=$ slope $^{*}$ gradient retention time + intercept obtained from the calibration line & \\
\hline $\log K$ IAM & $=0.29 * \mathrm{e}^{(0.026 \mathrm{CHI}[\mathrm{IAM}]+0.42)}+0.7$ & [42] \\
\hline Log k IAM & $=0.046 * \mathrm{CHI}(\mathrm{IAM})+0.42$ & [42] \\
\hline $\log \mathrm{K} H S A$ & $=\mathrm{e}^{\log \mathrm{k}(\mathrm{HSA})}$ & [42] \\
\hline $\log \mathrm{k} H S A$ & $=\log (\% \mathrm{HSA}$ bound $/[101-\% \mathrm{HSA}$ bound $])$ & [15] \\
\hline Estimated log Vdss & $=0.44 * \log \mathrm{K} \mathrm{IAM}-0.22 * \log \mathrm{K}$ HSA -0.62 & [42] \\
\hline Estimated log Vdu & $=0.23 * \log \mathrm{K} \mathrm{HSA}+0.43 * \log \mathrm{K} \mathrm{IAM}-0.72$ & [43] \\
\hline DEmax & $=100 / \mathrm{Vdu}$ & [11] \\
\hline Log k BTB & $=1.29 * \log \mathrm{k} I \mathrm{AM}+1.03 \log \mathrm{k}$ HSA -2.37 & [34] \\
\hline $\log k(P P B)$ & $=0.98 * \log \mathrm{kHSA}+0.19 * \log \mathrm{AGP}+0.031 * \mathrm{CHI} \log \mathrm{D} 7.4-0.20$ & {$[21]$} \\
\hline$\%$ BTB & $=100 * 10^{\log k \mathrm{BTB}} /\left(1+10^{\log \mathrm{k} \mathrm{B} B}\right)$ & [34] \\
\hline$\%$ PPB & $=100 * 10^{\log \mathrm{kPB}} /\left(1+10^{\log \mathrm{kPB}}\right)$ & [34] \\
\hline fu BTB and PPB & $=(100-\% \mathrm{BTB}) / 100$ and $(100 \%-\% \mathrm{PPB}) / 100$ & [34] \\
\hline $\mathrm{K}_{\mathrm{bb}}$ & $=$ fuPPB $/$ fuBTB & [34] \\
\hline Log Kpcell & $=1.1 \operatorname{logk} \mathrm{IAM}-1.9$ & [44] \\
\hline
\end{tabular}

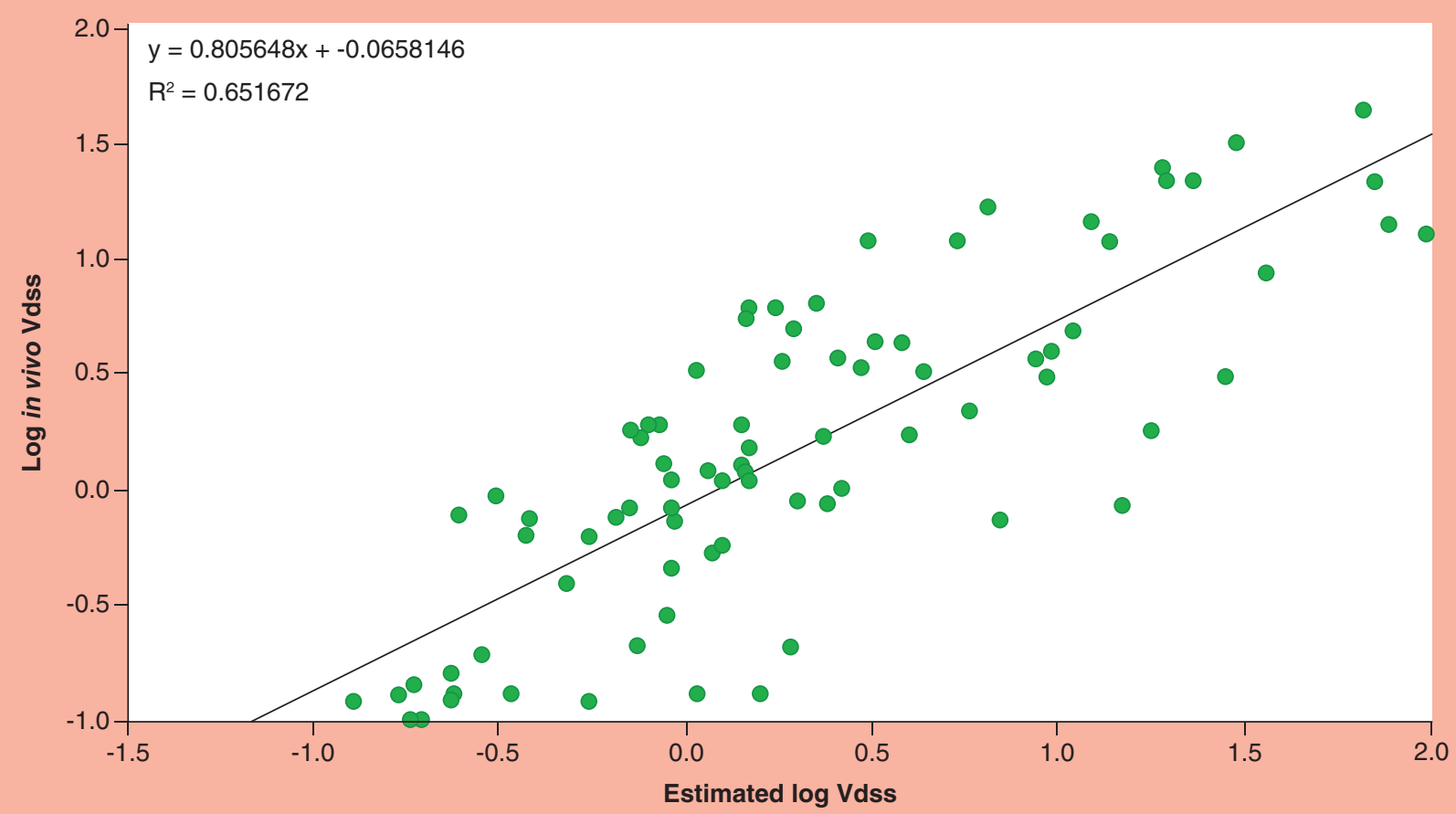

Figure 4. The measured and estimated human volume of distribution of known drugs. 
<smiles>COC(=O)C1=C(C)NC(C)=C(C(=O)OC)C1c1ccccc1[N+](=O)[O-]</smiles>

Nifedipine

$\mathrm{Vd}=0.7 \mathrm{l} / \mathrm{kg}$

$\mathrm{HSA} \%=88.8$

$\mathrm{CHI} \mathrm{IAM}=30.4$

$\log \mathrm{D} / \mathrm{P}=3.58$

$\mathrm{Cl}=9.8 \mathrm{ml} / \mathrm{min} / \mathrm{kg}$

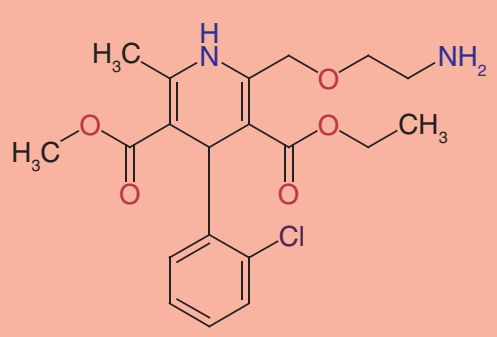

Amlodipine

$\mathrm{Vd}=21 \mathrm{l} / \mathrm{kg}$

HSA \% $=91.4$

$\mathrm{CHI} I \mathrm{AM}=51$

$\log \mathrm{D}=1.5 \log \mathrm{P}=3.01$

$\mathrm{Cl}=7 \mathrm{ml} / \mathrm{min} / \mathrm{kg}$

Figure 5. The physicochemical and biomimetic HPLC albumin (\% human serum albumin) and phospholipid (chromatographic hydrophobicity index immobilized artificial membrane) binding data together with the measured and estimated human in vivo volumes of distribution.

HSA: Human serum albumin.

that positively charged compounds bind more strongly to phospholipids and negatively charged membranes, while negatively charged compounds bind more strongly to albumin and have a low volume of distribution.

The volume of distribution model is a very good example of how to show that octanol/water log $\mathrm{D}$ values are not suitable to estimate tissue partition. Compounds that are charged at physiological $\mathrm{pHs}$ have a $\operatorname{lower} \log \mathrm{D}$, regardless of whether they have a positive or negative charge; the volume of distribution depends on the type of charge. Figure 5 shows two drug molecules and their $\log \mathrm{D} / \mathrm{P}$ values, as well as the biomimetic HPLC, HSA and IAM binding. The model, based on the measured albumin and phospholipid binding using chromatography, estimated well the big differences between the volume of distribution of the two compounds, Nifedipine and Amlodipine. Amlodipine has a positively charged basic group that significantly increases its phospholipid binding and, consequentially, the volume of distribution.

The unbound volume of distribution ( $\mathrm{Vdu}$ ) could be modeled by the sum of the two types of binding, as shown in Table 1 . The Vdu is proportional to the dose divided by the free plasma concentration. The sum of the two types of binding was shown to correlate better with the lipophilicity of the nonionized form of the molecules [43]. If the free-drug hypothesis is true and no significant active transport or permeability barrier exists, the reciprocal value of the Vdu equals the drug efficiency (DE) [11]. The DE is defined as the free biophase concentration relative to the dose, and therefore it is an important parameter to consider during the lead optimization process. It summarizes the nonspecific binding of the compound in the body as it reduces the free concentration that is available at the site of action. Therefore, compounds with low DE would require a higher dose. The DE is inversely proportional to the sum of the albumin and the phospholipid binding of the compound and inversely related to $\log P$.

The chromatographic protein and phospholipid-binding data are also suitable to estimate brain and lung tissue binding [34]. Depending on the proportion of the phospholipid and protein in various tissues, the weighted sums of the albumin and IAM binding can model the tissue binding. The mucus binding can be modeled by AGP binding as $90 \%$ of the mucus is glycoprotein that has similar binding characteristic to AGP. The brain to blood ratio can be estimated by using the models for the brain tissue binding and the plasma protein binding. The errors in these models are large as the errors are the sum of the errors from each individual model. However, it has been demonstrated that this method is able to differentiate between compounds that enter the CNS [34]. 


\section{Applications for new modalities, peptides \& macrocycles}

Another advantage of the chromatographic methods, beside their ability to model a compound's binding to the major body components (proteins and lipids), is that the method can be used for large molecules such as peptides, macrocycles and macrolides [46-49]. These large and amphiphilic molecules may accumulate at the solvent interface during octanol/water partition experiments, resulting in the bulk liquid concentration being practically zero in the octanol phase. The 3D structure and the conformational flexibility should be also taken into account when assessing their polarity and lipophilicity [50,51]. However, these molecules do bind to proteins and lipids in the body. Thus, the in silico calculated $\log$ P or $\log \mathrm{D}$ values are extremely low, while the measured chromatographic lipophilicity is much higher. It has been shown that macrolides can be characterized by biomimetic HPLC measurements $[46,47]$ and, for example, the large volume of distribution for erythromycin was successfully predicted by HPLC measurements. The cellular pharmacokinetics of macrolides has also been successfully predicted, indicating that compounds that were lipophilic and showed strong IAM binding, stayed in the cells longer [47]. Supercritical fluid chromatography has also been applied to measure the accessible polar surface area of macrocycles that can change their conformation depending on the polarity of the medium they are in [52].

The major obstacle to using biomimetic HPLC for characterizing large molecular weight compounds is that they interact with the stationary phase at multiple points and therefore, the binding kinetic is very slow producing wide chromatographic peaks. This makes retention time measurements less precise and compromises the detection limit of the compounds. At this stage, compounds with a molecular weight of around 2000 can be successfully characterized.

\section{Future perspective}

Big pharma companies are already using chromatographic techniques to measure properties of large number of drug discovery compounds in order to assess their chances to be developed as drugs [17,53]. Chromatographic methodology, especially with sensitive detection methods such as UV and mass spectrometry, can provide a fully automated and high-throughput measurement of a compound's lipophilicity and their binding to the major body components, albumin, glycoprotein and phosphatydil choline. New stationary phases containing other types of phospholipids will provide additional information on phospholipid binding in the future. The chromatographic technique offers versatility in this respect by developing new biomimetic stationary phases. When the compositions of the various tissues are known, simple models can be developed to measure tissue binding. Physiologically based pharmacokinetic modeling [54,55] approaches, which use calculated lipophilicity and pKa values of compounds for the models, can be significantly improved when measured tissue binding data are used.

\section{Executive summary}

- A large number of chromatography based biomimetic properties, such as protein and phospholipid binding data, have already been compiled in databases and numerous in vivo distribution models have been developed using these data, such as volume of distribution and various tissue bindings.

- Compound distribution depends on the difference between the binding to phospholipids and to proteins. The phospholipid binding is stronger for positively charged compounds; therefore, they partition more into tissues, while negatively charged compounds bind more strongly to albumin and prefer to stay in the plasma compartment.

- Octanol/water distribution coefficient is not able to differentiate between the type of charges on the molecules.

- The drug efficiency and the drug efficiency index can be evaluated using the chromatographic protein and phospholipid binding data much before any animal experiments and they can be used to prioritize compounds for further studies; this methodology is applicable for molecules that are beyond the rule of five property space, such as cyclic peptides, macrocycles and peptides.

- In the future, using the chromatographic peak width and peak shape information, there will be a possibility to derive the kinetic aspects of the binding of drug discovery compounds, including the protein targets, which will enable a more accurate estimation of pharmacokinetic behavior at a very early stage of the drug discovery process. 
Financial \& competing interests disclosure

The authors have no relevant affiliations or financial involvement with any organization or entity with a financial interest in or financial conflict with the subject matter or materials discussed in the manuscript. This includes employment, consultancies, honoraria, stock ownership or options, expert testimony, grants or patents received or pending, or royalties.

No writing assistance was utilized in the production of this manuscript.

\section{Open access}

This work is licensed under the Attribution-NonCommercial-NoDerivatives 4.0 Unported License. To view a copy of this license, visit http://creativecommons.org/licenses/by-nc-nd/4.0/

\section{References}

Papers of special note have been highlighted as: • of interest; $\bullet \bullet$ of considerable interest

1. Perola E. An analysis of the binding efficiencies of drugs and their leads in successful drug discovery programs. J. Med. Chem. 53(7), 2986-2997 (2010).

2. Cheng A, Diller DJ, Dixon SL, Egan WJ, Lauri G, Merz KM. Computation of the physio-chemical properties and data mining of large molecular collections. J. Comput. Chem. 23(1), 172-183 (2002).

3. Van de Waterbeemd H, Smith DA, Beaumont K, Walker DK. Property-based design: optimization of drug absorption and pharmacokinetics. J. Med. Chem. 44(9), 1313-1333 (2001).

- The fundamental paper that initiated the concept of property-based drug design. It highlighted the importance of the physicochemical properties of compounds that influence their fate in the body.

4. Collins I, Workman P. New approaches to molecular cancer therapeutics. Nat. Chem. Biol. 2(12), 689-700 (2006).

5. Hughes JD, Blagg J, Price DA et al. Physiochemical drug properties associated with in vivo toxicological outcomes. Bioorganic Med. Chem. Lett. 18(17), 4872-4875 (2008).

-• Analyzes the properties of large number of compounds and highlighted which properties show the highest probability of toxicological outcome.

6. Nava-Ocampo AA, Bello-Ramírez AM. Lipophilicity affects the pharmacokinetics and toxicity of local anaesthetic agents administered by caudal block. Clin. Exp. Pharmacol. Physiol. 31(1-2), 116-118 (2004).

7. Meanwell NA. Improving drug candidates by design: a focus on physicochemical properties as a means of improving compound disposition and safety. Chem. Res. Toxicol. 24(9), 1420-1456 (2011).

8. Ritchie TJ, Macdonald SJF. The impact of aromatic ring count on compound developability - are too many aromatic rings a liability in drug design? Drug Discov. Today 14(21-22), 1011-1020 (2009).

9. Valko KL, Teague SP, Pidgeon C. In vitro membrane binding and protein binding (IAM MB/PB technology) to estimate in vivo distribution: applications in early drug discovery. ADMET DMPK 5(1), 14-38 (2017).

10. Montanari D, Chiarparin E, Gleeson MP et al. Application of drug efficiency index in drug discovery: a strategy towards low therapeutic dose. Expert Opin. Drug Discov. 6(9), 913-920 (2011).

- Describes the drug efficiency concept that emphasize the importance of the free concentration of the drug at the site of action. When the drug efficiency is combined with the dose, it results in the drug efficiency index that can be used for ranking compounds.

11. Valko K, Chiarparin E, Nunhuck S, Montanari D. In vitro measurement of drug efficiency index to aid early lead optimization. J. Pharm. Sci. 101(11), 4155-469 (2012).

12. Arnott JA, Planey SL. The influence of lipophilicity in drug discovery and design. Expert Opin. Drug Discov. 7(10), 863-875 (2012).

- Provides an expert opinion about the importance of lipophilicity in drug discovery and design.

13. Zeliger HI. Exposure to lipophilic chemicals as a cause of neurological impairments, neurodevelopmental disorders and neurodegenerative diseases. Interdiscip. Toxicol. 6(3), 101-110 (2013).

14. Endo S, Goss KU. Serum albumin binding of structurally diverse neutral organic compounds: data and models. Chem. Res. Toxicol. 24(12), 2293-2301 (2011).

15. Valko K, Nunhuck S, Bevan C, Abraham MH, Reynolds DP. Fast gradient HPLC method to determine compounds binding to human serum albumin. relationships with octanol/water and immobilized artificial membrane lipophilicity. J. Pharm. Sci. 92(11), 2236-2248 (2003).

-. Describes the experimental procedures for the determination of human serum albumin binding of compounds using chemically bonded albumin stationary phases by high-performance liquid chromatography (HPLC). It also provides a comparison of protein and phospholipid binding with the octanol/water partition coefficients using the Abraham, solvation equation approach. It shows that for neutral compounds that octanol/water lipophilicity is a good model for albumin and phospholipid binding, but the charged compounds behave differently. 
16. Valko K, Ivanova-berndt G, Beswick P, Swindell WR, Kindey M, Ko D. Application of biomimetic HPLC to estimate lipophilicity, protein and phospholipid binding of potential peptide therapeutics. ADMET DMPK 6(2), 162-175 (2018).

- Describes the applicability of biomimetic HPLC property measurements for peptides as a representation of new modalities in drug discovery.

17. Bunally S, Young RJ. The role and impact of high throughput biomimetic measurements in drug discovery. ADMET DMPK 6(2), 74-84 (2018).

- Shows how the chromatographic property measurements of a large number of drug discovery compounds impact the lead optimization at GlaxoSmithKline.

18. Zheng X, Li Z, Beeram S et al. Analysis of biomolecular interactions using affinity microcolumns: a review. J. Chromatogr. B Analyt. Technol. Biomed. Life Sci. 968, 49-63 (2014).

- Summarizes the methods that can be used in affinity microcolumns for the determination of molecular interactions of compounds with proteins and potentially drug targets.

19. Chen S, Sobansky MR, Hage DS. Analysis of drug interactions with high density lipoprotein by high-performance affinity chromatography. Anal. Biochem. 397(1), 107-114 (2012).

20. Bi C, Beeram S, Li Z, Zheng X, Hage DS. Kinetic analysis of drug-protein interactions by affinity chromatography. Drug Discov. Today Technol. 17, 16-21 (2015).

21. Horváth C, Melander W, Molnár I. Solvophobic interactions in liquid chromatography with nonpolar stationary phases. J. Chromatogr. A 125(1), 129-156 (1976).

22. Vailaya A, Horváth C. Solvophobic theory and normalized free energies of nonpolar substances in reversed phase chromatography. J. Phys. Chem. B 101(30), 5875-5888 (1997).

23. Valkó K, Snyder LR, Glajch JL. Retention in reversed-phase liquid chromatography as a function of mobile-phase composition. J. Chromatogr. A 656(1-2), 501-520 (1993).

24. Janicka M, Kwietniewski L, Perišić-Janjić NU. Determination of retention factors of s-triazines homologous series in water using a numerical method basing on Ościk's equation. Chromatographia 63(Supp_13), S87-S93 (2006).

25. Janicka M. Application of Ościk's equation for description of solute retention in RP HPLC and calculation of retention factor in water. J. Liq. Chromatogr. Relat. Technol. 32(19), 2779-2794 (2009).

26. Valkó K. General Approach for the estimation of octanol/water partition coefficient by reversed-phase high-performance liquid chromatography. J. Liq. Chromatogr. Relat. Technol. 7(7), 1405-1424 (1984).

27. Du CM, Valko K, Bevan C, Reynolds D, Abraham MH. Rapid gradient RP-HPLC method for lipophilicity determination: a solvation equation based comparison with isocratic methods. Anal. Chem. 70(20), 4228-4234 (1998).

28. Valko K, Du CM, Bevan C, Reynolds DP, Abraham MH. Rapid method for the estimation of octanol/water partition coefficient (Log Poct) from gradient RP-HPLC retention and a hydrogen bond acidity term ( $\Sigma \alpha 2 \mathrm{H})$. Curr. Med. Chem. 8(9), 1137-1146 (2001).

29. Abraham MH. Scales of solute hydrogen-bonding: their construction and application to physicochemical and biochemical processes. Chem. Soc. Rev. 22(2), 73-83 (1993).

- Fundamental paper that describes the solvation equation approach to describe various solvent/solvent, chromatographic and biological distribution processes.

30. Canals I, Valkó K, Bosch E, Hill AP, Rosés M. Retention of ionizable compounds on HPLC. 8. Influence of mobile-phase pH change on the chromatographic retention of acids and bases during gradient elution. Anal. Chem. 73(20), 4937-4945 (2001).

31. Espinosa S, Bosch E, Rosés M, Valkó K. Change of mobile phase $\mathrm{pH}$ during gradient reversed-phase chromatography with 2,2,2-trifluoroethanol-water as mobile phase and its effect on the chromatographic hydrophobicity index determination. J. Chromatogr. A 954(1-2), 77-87 (2002).

32. Subirats X, Rosés M, Bosch E. On the effect of organic solvent composition on the $\mathrm{pH}$ of buffered HPLC mobile phases and the pKa of analytes - a review. Sep. Purif. Rev. 36(3), 231-255 (2007).

-• Details the theoretical background of the effects of the organic solvent compositions on the $\mathrm{pKa}$ of compounds and the $\mathrm{pH}$ of the chromatographic mobile phases.

33. Kaliszan R, Haber P, Tomasz B, Siluk D, Valko K. Lipophilicity and pKa estimates from gradient high-performance liquid chromatography. J. Chromatogr. A 965(1-2), 117-127 (2002).

34. Valko K. Physicochemical and biomimetic properties in drug discovery - chromatographic techniques for lead optimization. eBook First edition, 85-87 (2014).

35. Kratochwil NA, Huber W, Müller F, Kansy M, Gerber PR. Predicting plasma protein binding of drugs - revisited. Curr. Opin. Drug Discov. Devel. 7(4), 507-512 (2004).

-. Describes the theory of using chromatography with biomimetic stationary phases and the bases of standardization that enables the chromatographically derived property data to be reproducible and suitable for building databases. It also discusses the models for absorption and in vivo distribution of compounds using the measured data. 
36. Pidgeon C, Ong S, Liu H et al. LAM chromatography: an in vitro screen for predicting drug membrane permeability. J. Med. Chem. 38(4), 590-594 (1995).

- Pioneering work that described first the immobilized artificial membrane (IAM) chromatography using the stationary phase that was developed to emulate the lipid membrane of the cells.

37. McLaurin J, Chakrabartty A. Membrane disruption by Alzheimer beta-amyloid peptides mediated through specific binding to either phospholipids or gangliosides. J. Biol. Chem. 271(43), 26482-26489 (1996).

38. Casartelli A, Lanzoni A, Comelli R et al. A Novel and integrated approach for the identification and characterization of drug-induced cardiac toxicity in the dog. Toxicol. Pathol. 39(2), 361-371 (2011).

39. Peters TS. Do preclinical testing strategies help predict human hepatotoxic potentials? Toxicol. Pathol. 33(1), 146-154 (2005).

40. Casartelli A, Bonato M, Cristofori $\mathrm{P}$ et al. A cell-based approach for the early assessment of the phospholipidogenic potential in pharmaceutical research and drug development. Cell Biol. Toxicol. 19(3), 161-176 (2003).

- The first publication that showed relationship between IAM binding and phospholipidotic potential of compounds.

41. McDonnell PA, Caldwell GW, Masucci JA. Using capillary electrophoresis/frontal analysis to screen drugs interacting with human serum proteins. Electrophoresis 19(3), 448-454 (1998).

42. Hollósy F, Valkó K, Hersey A, Nunhuck S, Kéri G, Bevan C. Estimation of volume of distribution in humans from HPLC measurements of human serum albumin binding and immobilized artificial membrane partitioning. J. Med. Chem. 49(24), 6958-6971 (2006).

43. Valkó KL, Nunhuck SB, Hill AP. Estimating unbound volume of distribution and tissue binding by in vitro HPLC-based human serum albumin and immobilized artificial membrane-binding measurements. J. Pharm. Sci. 100(3), 849-862 (2011).

44. Gordon LJ, Allen M, Artursson P et al. Direct measurement of intracellular compound concentration by rapidfire mass spectrometry offers insights into cell permeability. J. Biomol. Screen. 21(2), 156-164 (2016).

- Shows the better correlation of IAM binding with measured intracellular concentration of compounds than with the calculated lipophilicity.

45. Young RJ, Green DVS, Luscombe CN, Hill AP. Getting physical in drug discovery II: the impact of chromatographic hydrophobicity measurements and aromaticity. Drug Discov. Today. 16(17-18), 822-830 (2011).

46. Stepanić V, Žiher D, Gabelica-Marković V et al. Physicochemical profile of macrolides and their comparison with small molecules. Eur. J. Med. Chem. 47(1), 462-472 (2012).

47. Stepanić V, Koŝstrun S, Malnar I et al. Modeling cellular pharmacokinetics of 14- and 15-membered macrolides with physicochemical properties. J. Med. Chem. 54(3), 719-733 (2011).

48. Valko K, Ivanova-berndt G, Beswick P, Swindell WR, Kindey M, Ko D. Application of biomimetic HPLC to estimate lipophilicity, protein and phospholipid binding of potential peptide therapeutics. ADMET DMPK 6(2), 162-165 (2018).

49. Valko KL, Kindy M, Evans J, Ko D. In vitro biomimetic HPLC and in vivo characterisation of GM6, an endogenous regulator peptide drug candidate for amyotrophic lateral sclerosis. ADMET DMPK 6(2), 176-189 (2018).

50. Guimaraes CR, Mathiowetz AM, Shalaeva M, Goetz G, Liras S. Use of 3D properties to characterize beyond rule-of-5 property space for passive permeation. J. Chem. Inf. Model 52(4), 882-890 (2012).

51. Rossi Sebastiano M, Doak BC, Backlund M et al. Impact of dynamically exposed polarity on permeability and solubility of chameleonic drugs beyond the rule of 5. J. Med. Chem. 61(9), 4189-4202 (2018).

52. Goetz GH, Philippe L, Shapiro MJ. EPSA: a novel supercritical fluid chromatography technique enabling the design of permeable cyclic peptides. ACS Med. Chem. Lett. 5(10), 1167-1172 (2014).

-• Describes a supercritical fluid chromatographic approach to measure polar surface area of cyclic peptides. This gained a lot of interest in designing permeable peptides and estimating permeability of new modalities with special emphases on potential intramolecular hydrogen bonding.

53. Goetz GH, Shalaeva M. Leveraging chromatography based physicochemical properties for efficient drug design. ADMET DMPK 6(2), 85-104 (2018).

54. Poulin P, Dambach DM, Hartley DH et al. An algorithm for evaluating potential tissue drug distribution in toxicology studies from readily available pharmacokinetic parameters. J. Pharm. Sci. 102(10), 3816-3829 (2013).

55. Jusko WJ. Moving from basic toward systems pharmacodynamic models. J. Pharm. Sci. 102(9) 2930-2940 (2013). 
